\title{
Editorial
}

\section{Und sie bewegt sich doch}

Als ich unlängst den neuen Bundesabfallwirtschaftsplan durchsah, wurde ich durch einige Zahlen zum Stand der heutigen Abfallverbrennung an meine Anfangsjahre als Assistent am Institut von Professor Paul Brunner an der TU Wien erinnert. Damals durfte ich an der sogenannten ASTRA-Studie (Auswirkungen unterschiedlicher Szenarien der thermischen Verwertung von Abfällen in Österreich) mitwirken. Wesentliche Ergebnisse dieser Studie waren, dass das Aufkommen der brennbaren Abfälle damals (die Studie erschien 1997 mit Daten aus 1995) auf ca. 8,5 Mio t/a geschätzt wurde und für ca. 80 Abfall-Schlüsselnummern die optimalen Behandlungswege auf Basis ihrer stofflichen Zusammensetzung ermittelt wurden. Demnach sollten von den 8,5 Mio t/a rund 4,9 Mio t/a thermisch behandelt werden, während damals tatsächlich nur etwa 2,0 Mio t/a diesen Weg gingen, davon lediglich 0,5 Mio t/a in Müllverbrennungsanlagen. Heute, 20 Jahre später, werden rund 4,4 Mio t/a verbrannt. Davon 2,6 Mio t/a in Müllverbrennungsanlagen und 1,8 Mio t/a in anderen thermischen Behandlungsanlagen (z.B. den sogenannten Mitverbrennungsanlagen). Die ASTRA-Zahlen haben sich damit als ziemlich treffsicher erwiesen. Trotzdem muss ich feststellen, dass die Präsentationen der ASTRAStudie damals alles andere als angenehm waren. Unsere Kritiker warfen uns vor, unreflektiert der thermischen Verwertung das Wort zu reden. Dass die mechanisch-biologische Behandlung im optimierten Szenario nicht mehr aufschien, war Vielen ebenfalls ein Dorn im Auge. Aber auch hier hat die Zeit der ASTRA-Studie insofern Recht gegeben, da die Bedeutung der mechanisch-biologischen Behandlung in Österreich eher abnimmt.

Was ich für mich daraus mitnehme, ist Folgendes: Erstens, dass sich die Situation der Behandlung der brennbaren Abfälle in Österreich zum Positiven entwickelt hat. Dies ist zum Großteil der Deponieverordnung geschuldet, die aus meiner Sicht einen ganz wichtigen Schritt zur Umsetzung der AWGZiele darstellt. Es dauert eben einige Zeit, bis man solche Veränderungen und Erfolge sieht, und man darf nicht ungeduldig werden, wenn es einem nicht rasch genug vorwärts geht. Zweitens, dass es gut ist, wenn man Zielzustände technisch-naturwissenschaftlich sauber herleitet, auch wenn das die eine oder andere Stakeholder-Gruppe überfordert. Man muss versuchen, durch Sachargumente zu überzeugen. Die ASTRA-Studie wurde meines Wissens methodisch nie infrage gestellt. In meiner Einschätzung hätte sie sogar noch heute das Potenzial, die Zuordnung der brennbaren Abfälle zu den diversen Verbrennungsanlagen hinsichtlich Gesamtemissionen und Schadstofflenkung weiter zu optimieren.

Eine weitere, aus meiner Sicht sehr richtige Entwicklung, hat der Behandlungsgrundsatz zum Thema Klärschlamm im neuen Bundesabfallwirtschaftsplan genommen. Dort wird als Ziel für 2030 vorgegeben, dass 65 bis $85 \%$ des in Österreich anfallenden kommunalen Klärschlamms einer Phosphorrückgewinnung zugeführt werden sollen. Diese Zielvorgabe soll anhand einer technisch-naturwissenschaftlichen Studie, welche die Struktur der österreichischen Abwasserbehandlung und die technischen Gegebenheiten der heute verfügbaren Technologien des Phosphorrecyclings berücksichtigt, genauer festgemacht werden. Wie auch beim früher "heißen Thema Abfallverbrennung“ ist damit eine Basis geschaffen, um sachlich fundierte Zielvorgaben entwickeln zu können. Aus meiner ASTRA-Erfahrung bin ich heute guter Dinge, dass in 20 Jahren in Österreich ein effektives Phosphorrecycling umgesetzt ist. Die ersten ökonomischen Abschätzungen zeigen, dass dies durchaus leistbar ist.

Die Abfallwirtschaft bewegt sich also doch, und sie wird sich weiter in die richtige Richtung bewegen. Man muss nur lange genug dabei sein, um es zu bemerken. 
Univ.-Prof. DI Dr. H. Rechberger ( $ه$ )

Institut für Wassergüte und

Ressourcenmanagement, Technische

Universität Wien,

Karlsplatz 13/226,

1040 Wien, Österreich

helmut.rechberger@tuwien.ac.at 